\title{
Three-Dimensional Finite Element Simulations of The Rolling of Superconducting Wire
}

\author{
M. A. Davies \\ D. E. Boyce \\ P. R. Dawson
}

17 November 1993

\section{Introduction}

The goal of this work was to show the utility of using finite-element simulations to ascertain the quality of superconducting tape, created by the repeated rolling of an initially round wire. Steady-state simulations of the the rolling reductions of a twomaterial superconducting wire were performed using ISAIAH, a three-dimensional nonlinear finite-element code written by Dewhurst et. al. [1]. The simulations were done in conjunction with Dave Korzekwa at Los Alamos National Laboratory to produce results that could be compared with experimental findings. Results indicate that ISAIAH is capable of producing potentially useful data. but further work is necessary to obtain meaningful quantitative comparisons between simulations and experiments. This report describes the simulations that have been conducted to date and also indicates several directions for the future development of this research.

\section{Problem Description}

The initial workpiece was a $1 \mathrm{~mm}$ diameter, round wire consisting of an inner core of superconducting material and an outer sheath of silver. For these simulations, the 


\section{DISCLAIMER}

This report was prepared as an account of work sponsored by an agency of the United States Government. Neither the United States Government nor any agency thereof, nor any of their employees, make any warranty, express or implied, or assumes any legal liability or responsibility for the accuracy, completeness, or usefulness of any information, apparatus, product, or process disclosed, or represents that its use would not infringe privately owned rights. Reference herein to any specific commercial product, process, or service by trade name, trademark, manufacturer, or otherwise does not necessarily constitute or imply its endorsement, recommendation, or favoring by the United States Government or any agency thereof. The views and opinions of authors expressed herein do not necessarily state or reflect those of the United States Government or any agency thereof. 


\section{DISCLAIMER}

Portions of this document may be illegible in electronic image products. Images are produced from the best available original document. 


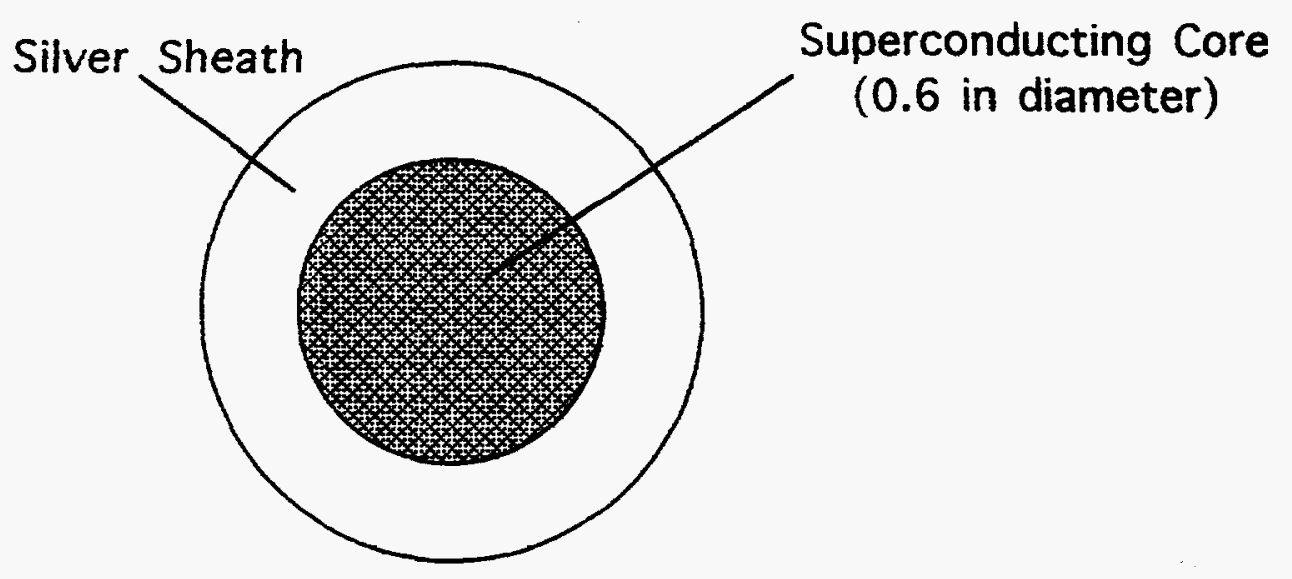

Wire Diameter: $1 \mathrm{~mm}$

Figure 1: Cross-section of superconducting wire workpiece.

wire had a $36 \%$ fill of superconductor corresponding to a core diameter of $0.6 \mathrm{~mm}$. The wire cross-section is shown in Figure 1.

The wire was reduced to a thin tape by repeated symmetric rolling reductions, using two $38.0 \mathrm{~mm}$ diameter rolls as shown in Figure 2. The roll speed at the contact surface was $5 \mathrm{~mm} / \mathrm{s}$. Friction between the roll and the workpiece was reported to be very high; therefore in these simulations a sticking constraint was adopted.

Figure 2 illustrates several of the issues that are pertinent to this problem. First, the workpiece consisted of two-materials. Although ISAIAH is capable of handling two-material simulations, changes in the mesh generator were required to make the implementation of the two-material problem easier. These changes are detailed in the Appendix.

The second issue is the calculation of the final shape of the workpiece. Because the initial mesh geometry was only a guess of the final deformed shape, corrections in the shape were made in accordance with the calculated velocity field. In typical homo- 


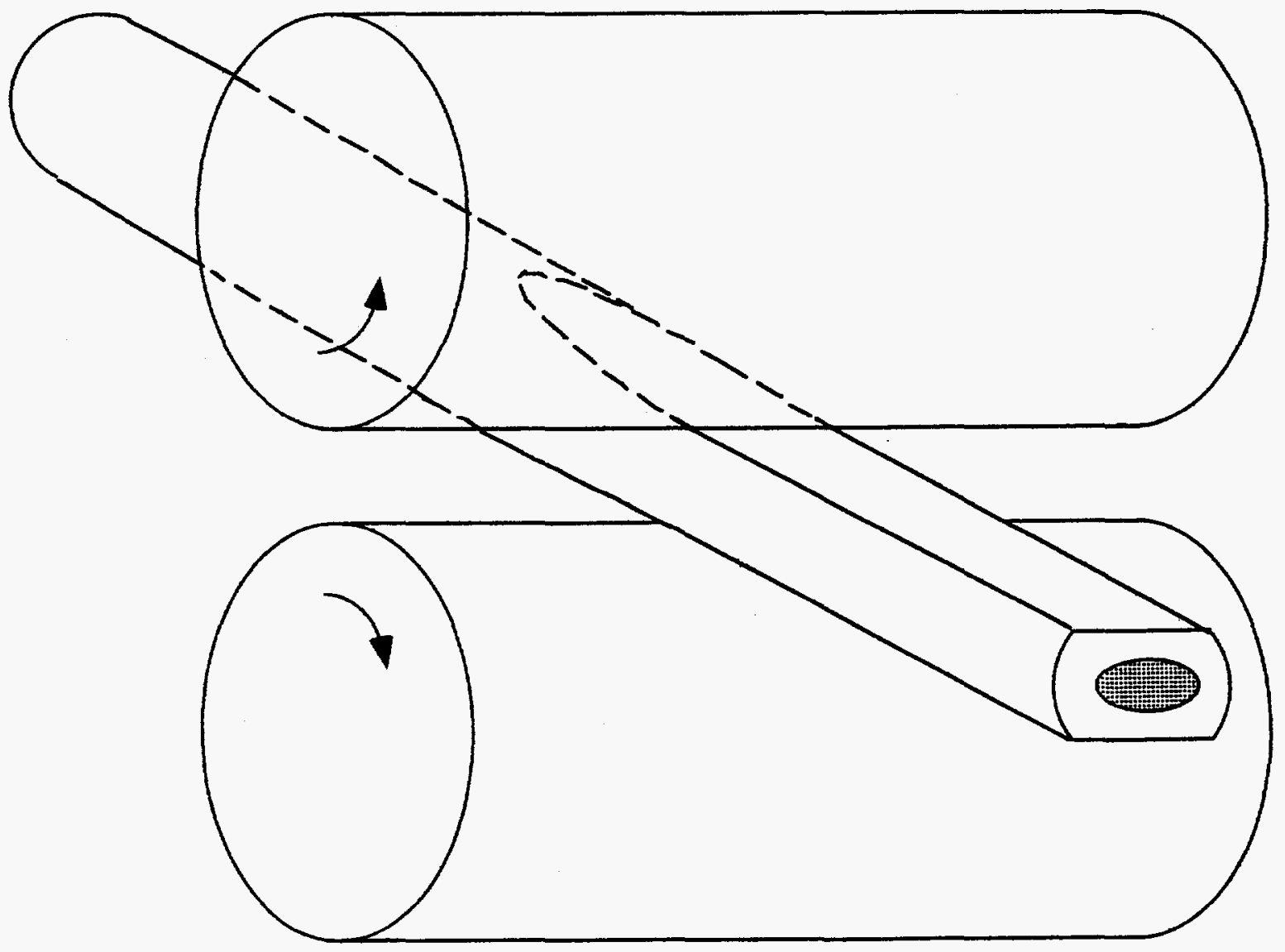

Figure 2: Schematic of the first rolling reduction. The relative sizes of objects have been altered to improve clarity. 
geneous rolling problems, only the external, traction-free, surfaces require correction. In ISAIAH, this is done by free-surface correction routines that adjust the shape of the workpiece to coincide with a stream surface of the material flow. However, for the two material case, the internal interface must also be adjusted. With only minor program changes, we were able to use the existing correction routines to solve the internal interface correction problem.

The third issue is the generation of mesh geometries for successive rolling passes. For each consecutive pass, an appropriate mesh must be generated using the final corrected geometry from the previous pass. A program entitled meshpost was developed to perform this task automatically. In these simulations, the first two meshes were generated manually while the others were generated using meshpost.

Another important problem is the distortion of the mesh geometry. During numerous successive rolling passes, the cumulative surface corrections can become quite extreme. These large corrections place limitations on the number and size of the elements that can be used in simulations. If meshes are too fine, surface corrections can cause severe distortion or even inversion of the elements. rendering further computation impossible. To alleviate this problem, routines were developed that smooth or average nodal point coordinates between surface corrections. Although these routines were not fully implemented during the course of this work, they will be available in later versions of ISAIAH. Use of these routines is crucial if more accurate quantitative data is desired.

\section{Rolling Simulations}

In this section. the results of the of the first two $15 \%$ rolling reductions of the superconducting wire are discussed. Details of program input and operation are included in the Appendix. 


\subsection{Mesh Generation}

To start the series of rolling reductions, it was necessary to generate a mesh for the initial rolling pass. Because of the four-fold symmetry of the problem, only one-quarter of the wire was considered. The roll contact line was taken to be the geometric intersection of the roll cylinder with the cylinder of the wire as shown in Figure 3. The radii of the wire and the roll were denoted $r_{1}$ and $r_{2}$, respectively. The roll axis intersected the $(x, z)$-plane at the point $\left(x_{0}, z_{0}\right)$ and the reduction fraction was denoted $f$ ( $f=0.15$ for a $15 \%$ reduction). It was assumed that the rolled wire departed from the roll tangentially at its base. The roll contact line was given by the set of points $(\hat{x}, \hat{y}, \hat{z})$ that formed the intersection of the wire and roll cylinders.

Using these definitions and assumptions, a mathematical expression for the roll contact line was derived. The range of contact between the roll and wire is an interval of the $x$-axis, $\left[\hat{x}_{1}, \hat{x}_{2}\right]$, defined by

$$
\begin{aligned}
& \hat{x}_{1}=x_{0}-\sqrt{r_{2}^{2}-\left(z_{0}-r_{1}\right)^{2}} \\
& \hat{x}_{2}=x_{0} .
\end{aligned}
$$

In this interval, the values of $\hat{y}$ and $\hat{z}$ are given by the following expressions.

$$
\begin{aligned}
& \hat{z}=z_{0}+\sqrt{r_{2}^{2}-\left(x-x_{0}\right)^{2}} \\
& \hat{y}=-\sqrt{r_{1}^{2}-\hat{z}^{2}}
\end{aligned}
$$

An initial guess for the shape of the mesh was generated using Equations 1 and 2 . However, difficulties arose at the point of first contact between the roll and the workpiece. The cause of the difficulty was the initiation of contact at a single point, rather 


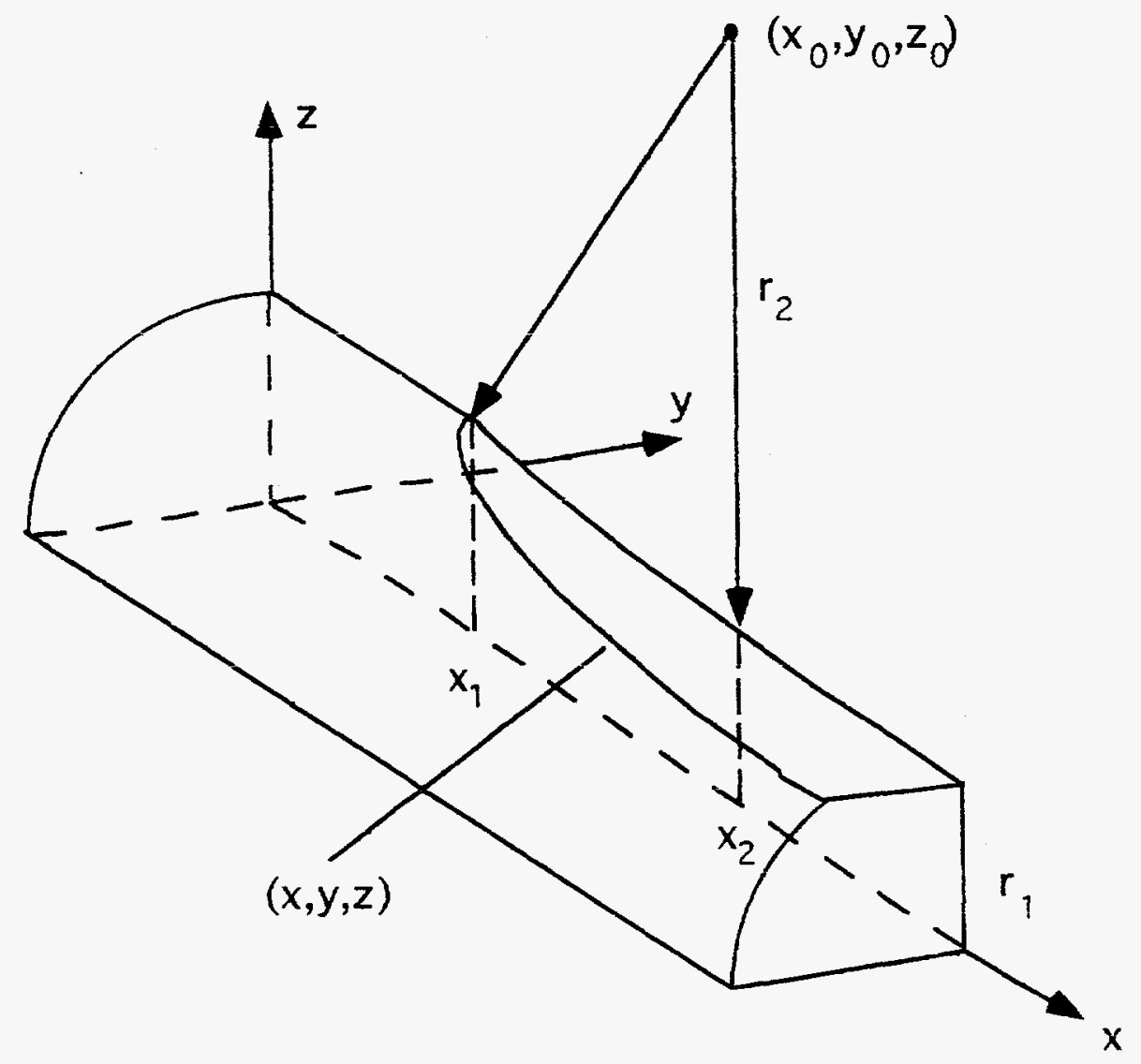

Figure 3: Geometry of the roll contact line for the first pass. 
Table 1: Table showing the surfaces declared for the rolling mesh.

\begin{tabular}{|l|l|}
\hline Surface Number & Description \\
\hline \hline 1 & Inlet Cross-section \\
\hline 2 & (x,y) Symmetry Plane \\
\hline 3 & Traction-free Surface Before Roll \\
\hline 4 & (x,z) Symmetry Plane \\
\hline 5 & Roll Contact Surface \\
\hline 6 & Traction-free Flat Surface After Roll \\
\hline 7 & Outlet Cross-section \\
\hline 8 & Traction-free Round Surface After Roll \\
\hline 9 & Internal Interface After Roll \\
\hline 10 & Internal Interface Before Roll \\
\hline
\end{tabular}

than along a curve as would be the case for a rectangular workpiece. This geometry could only be approximated with the available mesh generator. The approximation consisted of assuming that contact was initiated along a very short line segment rather than at a single point (see Figure 4). This difficulty did not arise for successive passes and hence the approximation probably had little effect on the overall results.

The mesh for the first rolling pass is shown in Figure 4. This mesh had 312, 20node quadralateral elements with a total of 1693 nodal points. On any cross-section, the outer seven elements made up the silver sheath. while the inner nineteen made up the superconducting core. In total, there were 84 elements in the silver sheath and 228 in superconducting core. The elements were made more dense on the roll contact surface where more rapid changes in the solution could be expected.

Ten surfaces were defined for use in specifying boundary conditions and surface corrections. These are summarized in Table 1. The total number of surface elements was 352. Surfaces 6.8 and 9 were subject to correction, the details of which are described in the Appendix. The number of surface elements subject to correction was 100. 


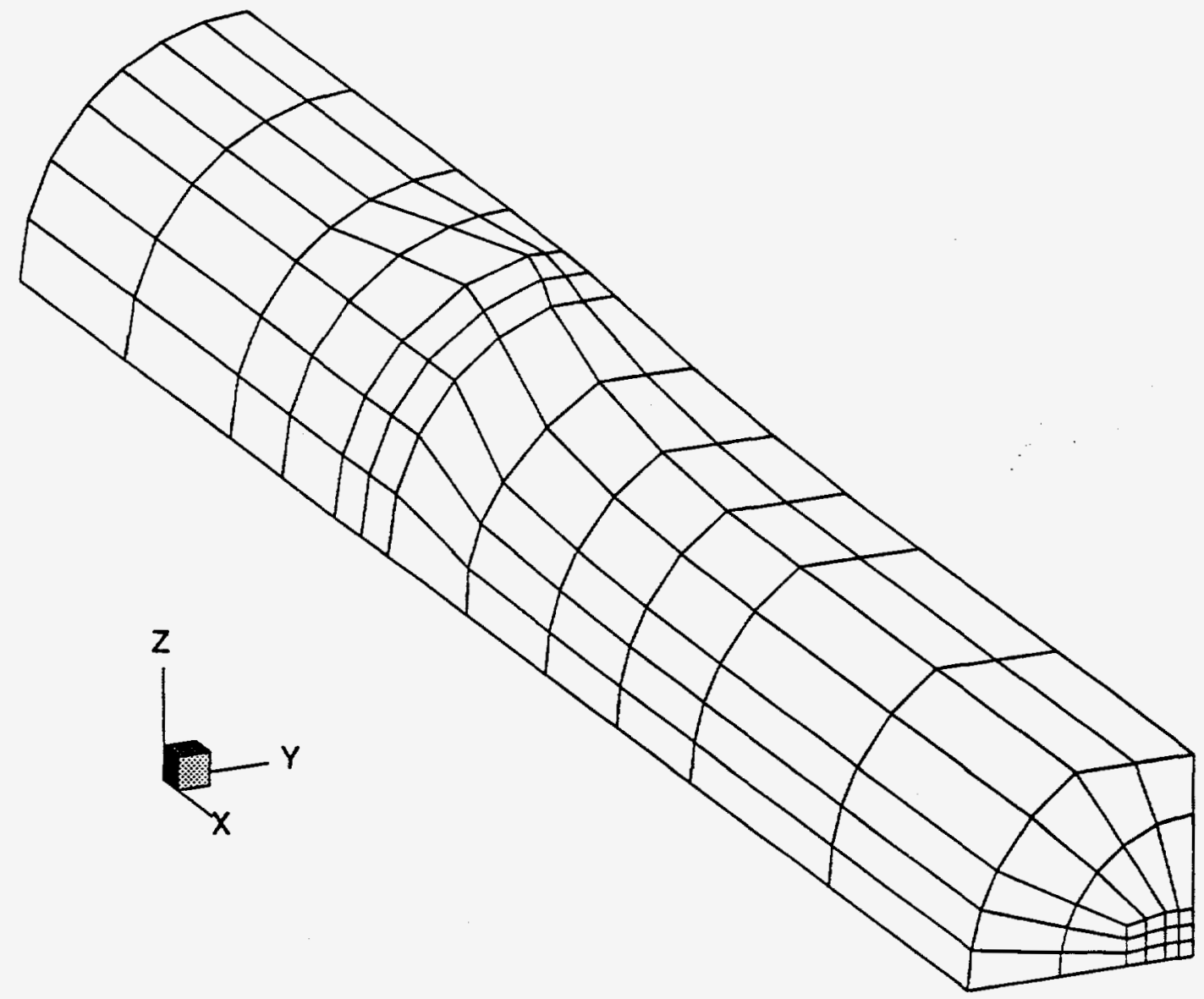

Figure 4: Initial mesh for the first rolling reduction of the superconducting wire. 


\subsection{Material Behavior}

Nonlinear material behavior was simulated by a simple non-evolving power law model ${ }^{1}$ of the form.

$$
\sigma_{I I}^{\prime}=A \mathrm{e}^{\frac{Q}{R \theta}}\left(d_{I I}^{\prime}\right)^{n}
$$

where $Q$ is the activation energy, $R$ is the universal gas constant and $\theta$ is the temperature. For these simulations the activation energy was zero, and hence the exponential term was 1.0. Therefore, the constants $A$ and $n$ defined the nonlinear material behavior. For the silver.

$$
\begin{gathered}
A_{s i l}=150 \mathrm{MPa} \\
n_{s i l}=0.02
\end{gathered}
$$

while for the superconductor,

$$
\begin{gathered}
A_{\text {sup }}=400 \mathrm{MPa} \\
n_{\text {sup }}=0.02 .
\end{gathered}
$$

The rate sensitivities were taken to be the same for the two materials. but the superconductor was considered to be much more resistant to deformation than the silver.

\subsection{Simulation Results}

Steady state solutions to the nonlinear viscoelastic rolling problem were generated using ISAIAH. Representative results of the first two rolling passes are discussed in this section.

For the first pass, ISAIAH produced the results shown in Figures 5,6 and 7 . Figure 5 shows a contour of the $\mathrm{x}$-velocity profile and Figures 6 and 7 show contours

\footnotetext{
${ }^{1}$ More sophisticated power law models containing an evolving state variable have been used to describe the two materials in two-dimensional simulations. However, a state variable-power law model is not currently available on ISAIAH. Other state variable models such as Kocks or Hart's model are available. but the proper parameter values for these model would have to be determined.
} 
of the $y$ - and z-velocities. respectively. The negative $y$-velocity concentration just beneath the roll was the main driver for the correction of the external free-surface. This concentration occurred much higher on the workpiece (larger $\mathrm{z}$ value) than it did for a homogeneous wire. This indicated that the silver was being squeezed out over the harder inner core, causing a noticeable squaring-off of the workpiece final crosssection. Correction of the internal cross-section also caused significant oblateness in the initially round cross-section of the superconductor. In general, the results are in keeping with our physical intuition about the problem.

The final cross-sectional geometry from the first pass was used to manually generate a mesh for the second pass. For all succeeding passes, meshes were generated by the program meshpost as described in the Appendix. The results from the third pass are shown in Figures 8,9 and 10. Again the free-surface correction was driven by the concentration of y-velocity. However, this concentration has now moved to a lower point on the workpiece. For this pass, the correction of both the internal and external geometries became more pronounced: significant flattening of the workpiece has occurred. leaving a nearly rectangular final cross-section.

Five rolling passes were completed in total. Results from the fifth and final pass are shown in Figures 11, 12 and 13. The qualitative characteristics of the velocity fields shown in these plots are similar to those of the previous passes, however the mesh has now undergone substantial deformation. The final outer cross-section is rectangular, but the core cross-section still retains significant curvature.

\section{Conclusions}

This work has demonstrated the ability of ISAIAH to simulate the rolling reduction of a two-material, superconducting wire. The cumulative effect of the repeated rolling was to reduce the z-dimension of the wire from $1 \mathrm{~mm}$ to approximately $0.38 \mathrm{~mm}$. 


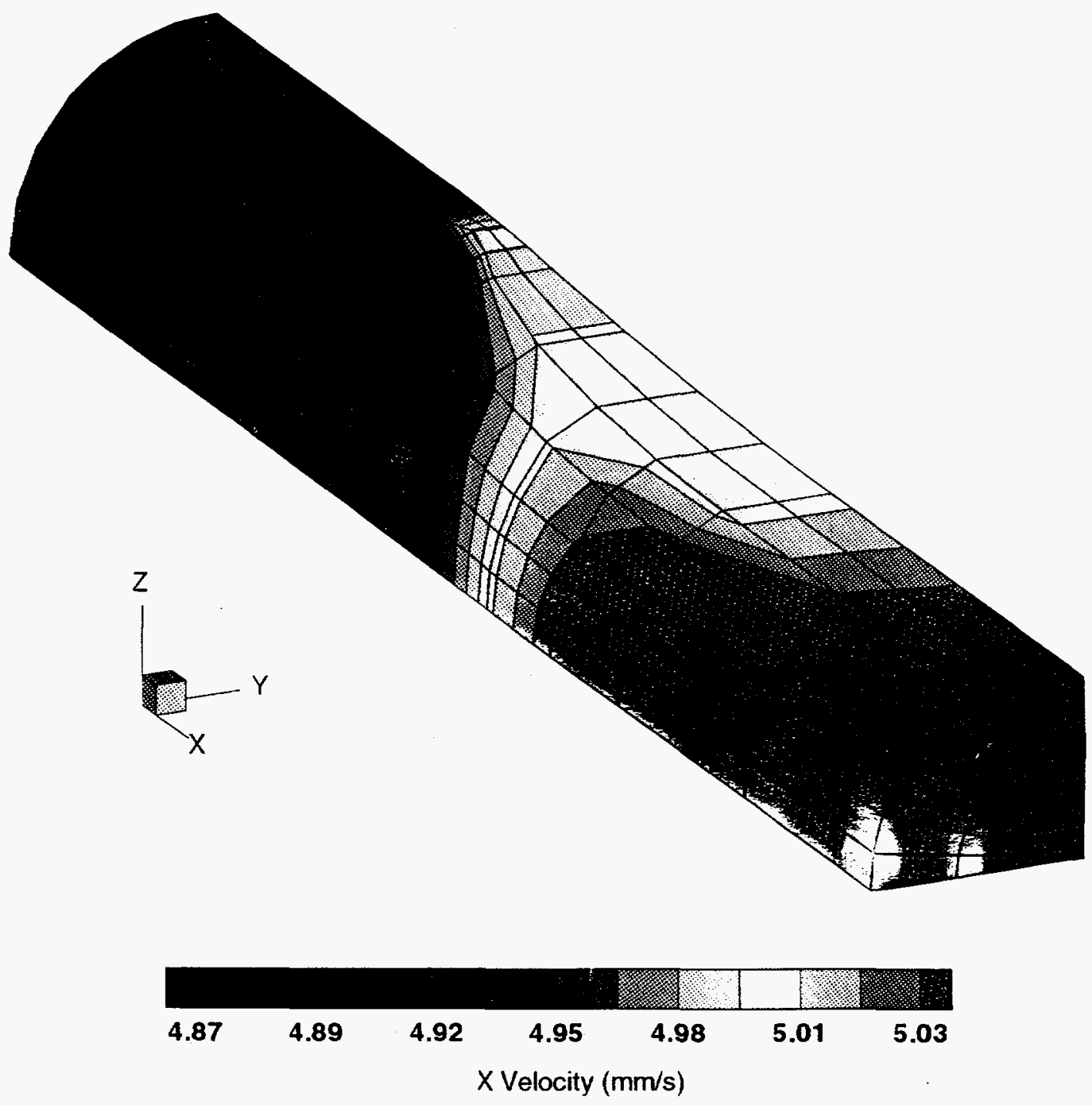

Figure 5: Contour of $\mathrm{x}$-velocity for the first reduction. 


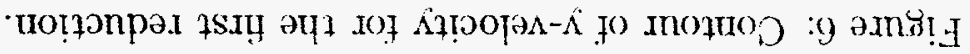

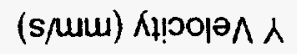

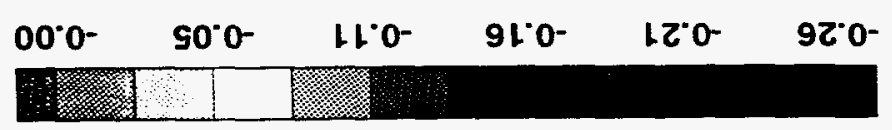

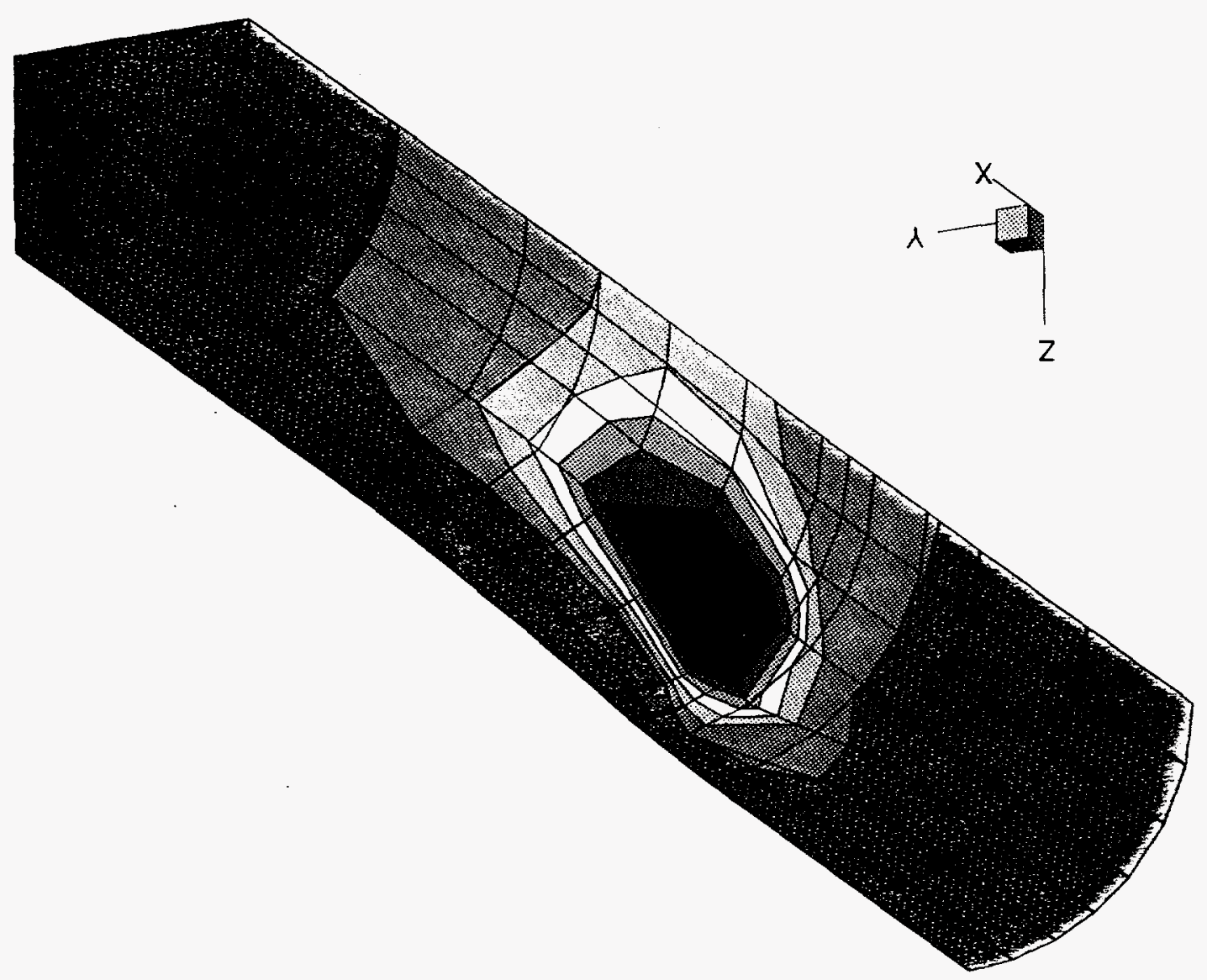



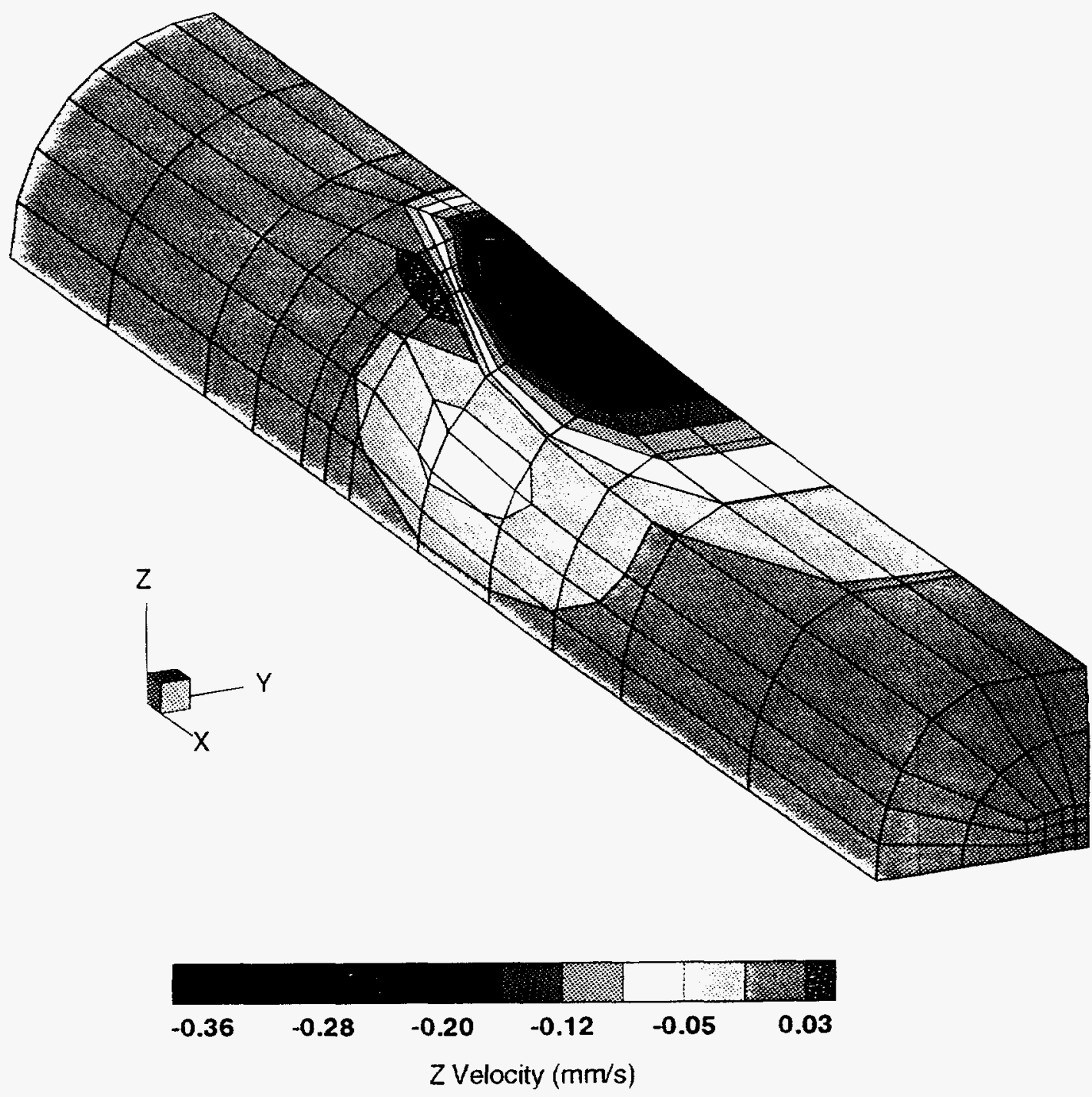

Figure $\mathrm{T}$ : Contour of $z$-velocity for the first reduction. 


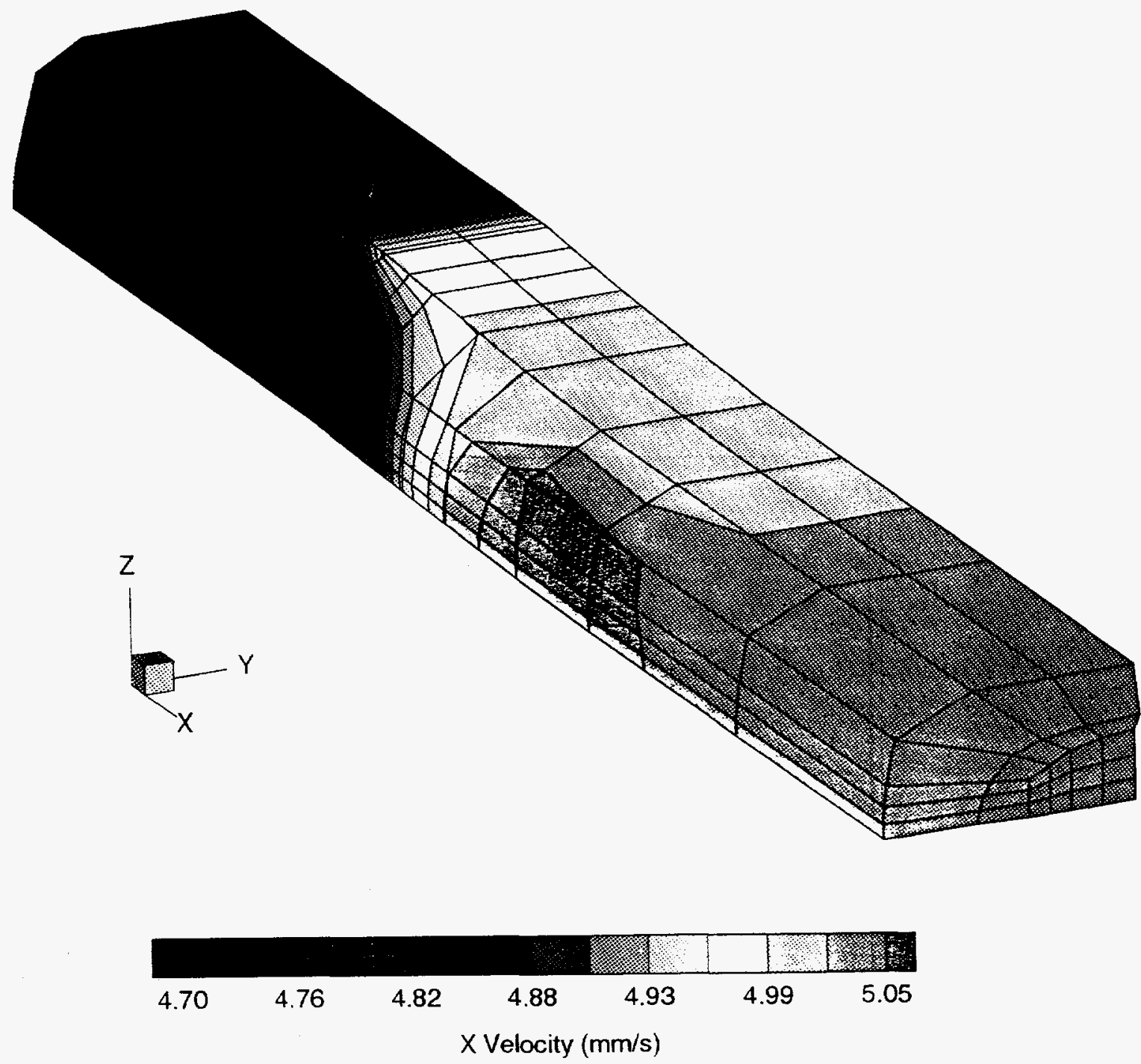

Figure $\mathrm{x}$ : Contour of $\mathrm{x}$-velocity for the third reduction. 


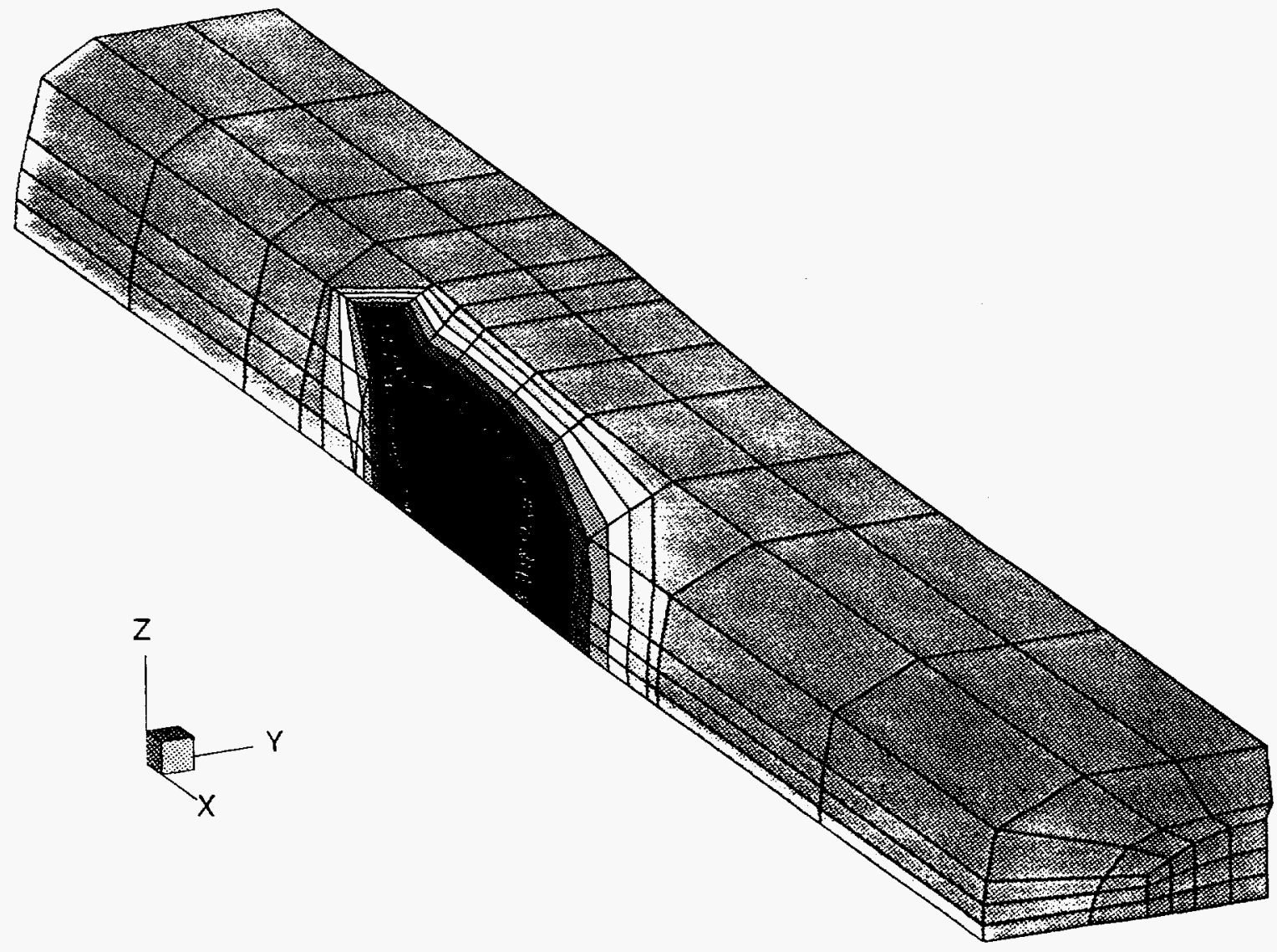

\begin{tabular}{lllllll}
\hline & & & & & & S \\
\hline-0.23 & -0.18 & -0.13 & -0.09 & -0.04 & 0.00 \\
& & & $Y$ Velocity $(\mathrm{mm} / \mathrm{s})$
\end{tabular}

Figure 9: Contour of v-velocity for the third reduction. 

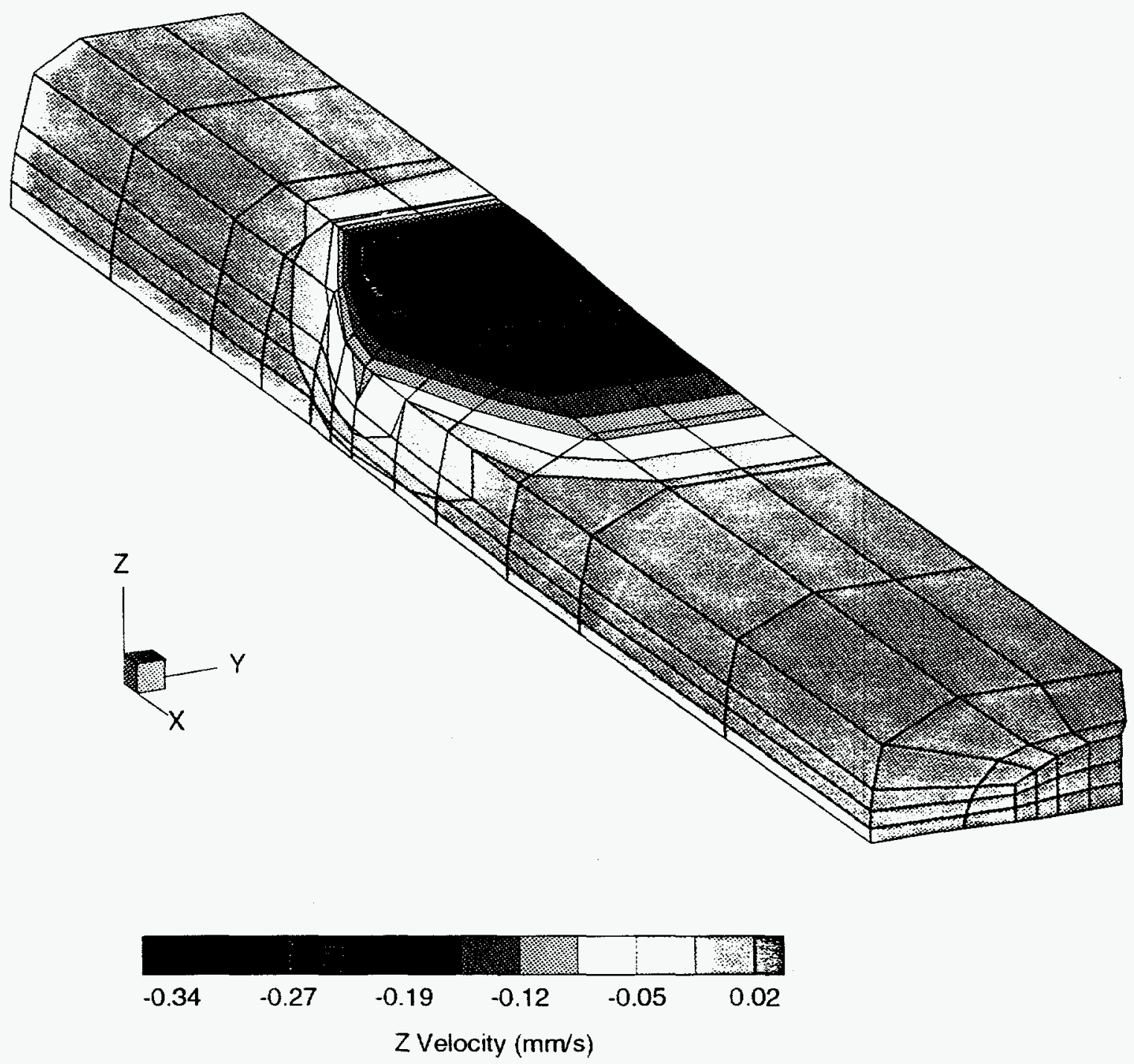

Finure 10: Contour of "z-velocity for the third reduction. 


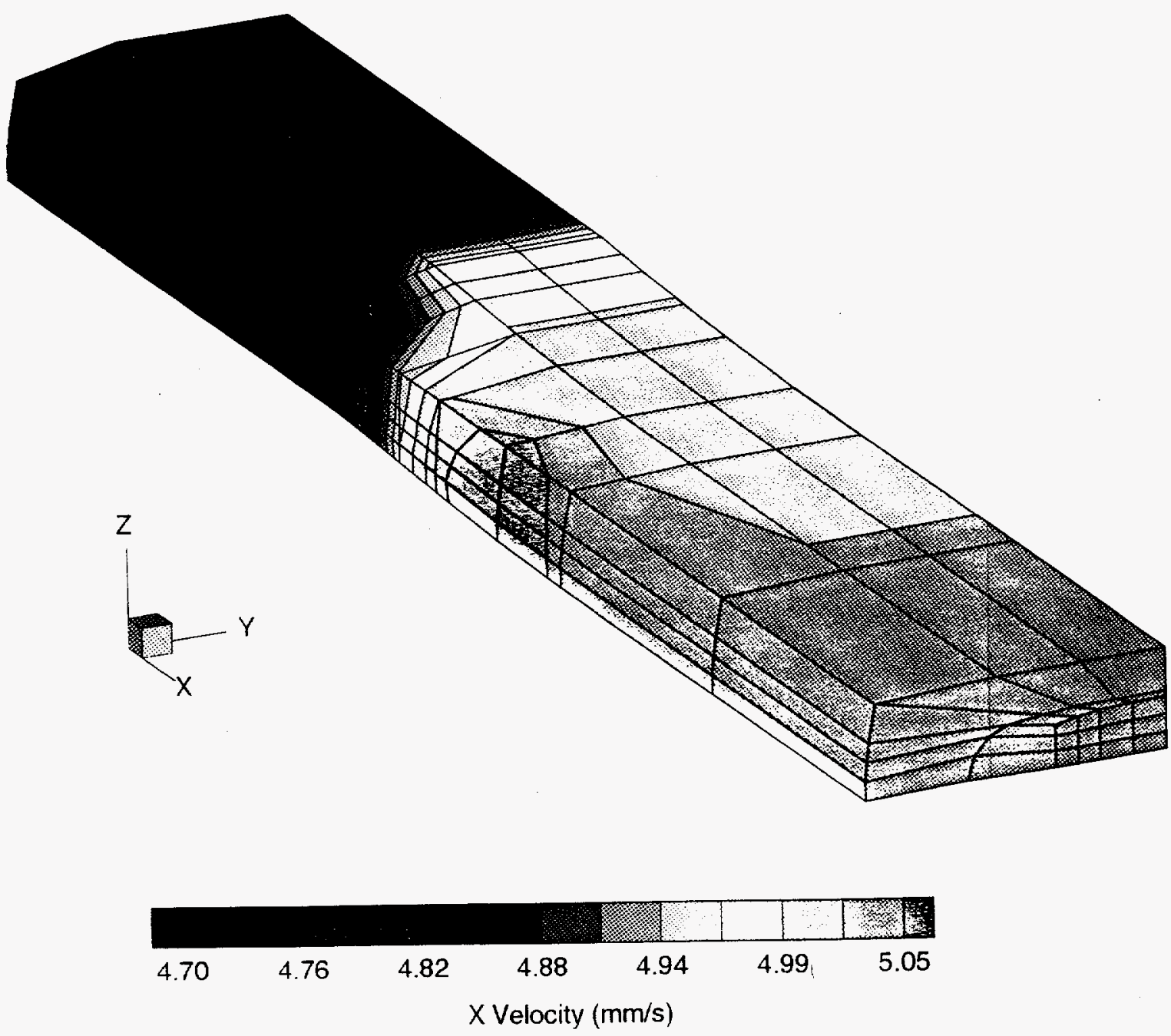

Figme 11: Contom of x-velocity for the fifth rectuction. 


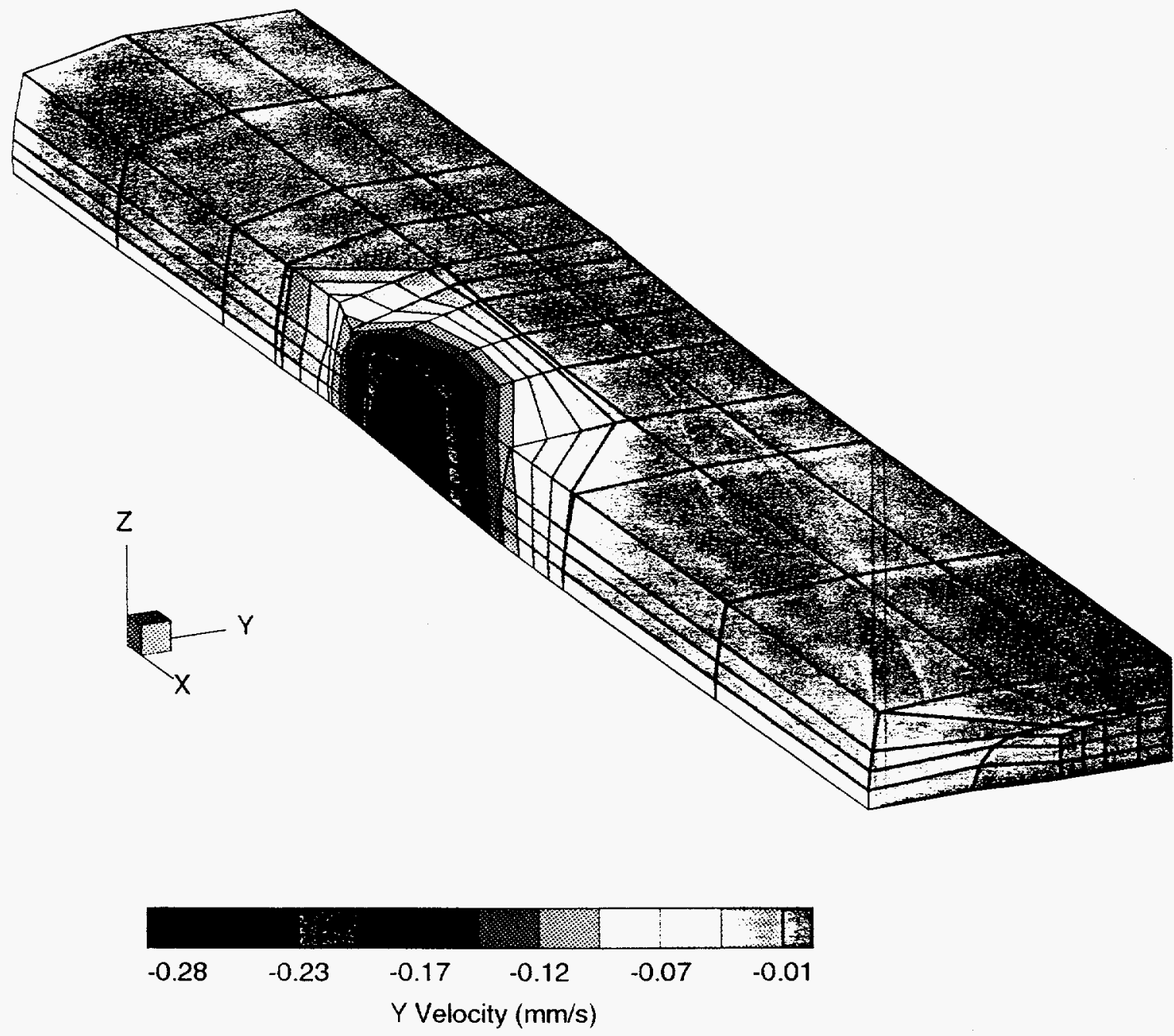

Figne 12: Contour of v-velocity for the lifth reduction. 


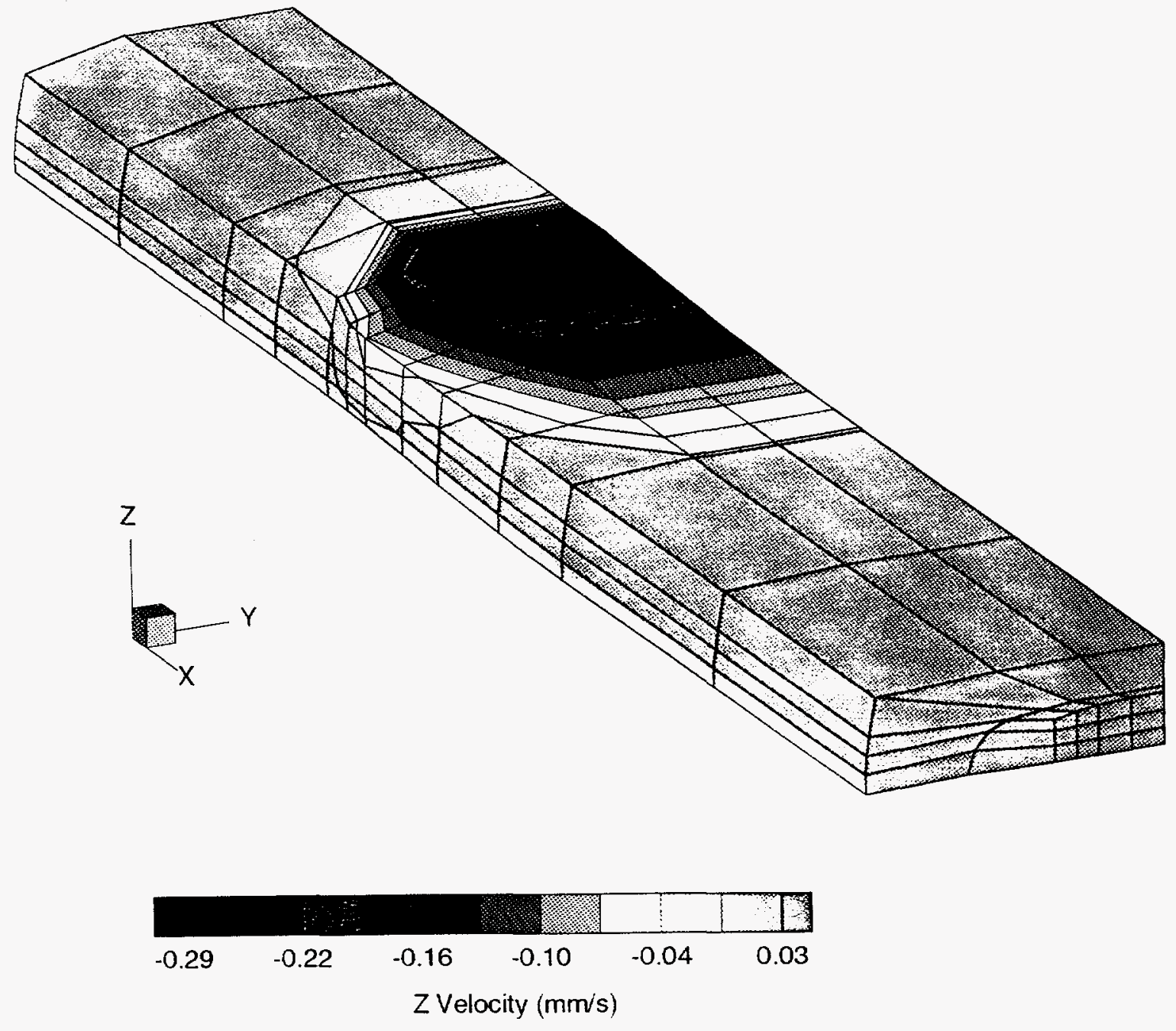

Fignue 13: Contone of $\%$-redocity tor the lifth reduction. 
Although this drastic total reduction caused a marked distortion of the elements of the mesh, the program was still quite able to perform the necessary calculations. Indeed, further reductions would be possible if the mesh geometry was re-evaluated to take advantage of the nearly rectangular, deformed shape of the wire.

The promise of these initial results justifies future work in this area. Some of the possible directions this work might take in the future are summarized below.

- Develop a power-law model for ISAIAH that incorporates an evolving state variable. The simulations could then take advantage of data on material behavior available from Los Alamos. An alternative to this is to attempt to fit data from experiments with other state-variable models available in ISAIAH.

- Allow the superconducting core to have some compressibility. Data from experiments suggest that effect is very important in determining the final shape of the wire.

- Develop new surface correction routines for ISAIAH that solve the problem by integrating along characteristic curves. Galerkin techniques for solving the freesurface problem have proved troublesome for this problem. Solution techniques which reduce the problem to integrals along characteristic curves may eliminate some problems associated with Galerkin techniques. For example, the definition of boundary conditions becomes much easier when the problem is solved along characteristics.

- Use the calculated velocity fields from ISAIAH to determine the orientation history of crystals in the superconducting core. This is actually the crux of the problem since it is the orientation of these crystals that determines the superconducting properties of the core. 


\section{A Details of the Simulations}

\section{A.1 Mesh Generator}

The mesh generator, mesh3d.v2, was used to generate the meshes for these simulations. This mesh generator requires an input file that defines the mesh geometry in terms of interconnected 20-point quadrilaterals called regions (see Appendix). Accurate representation of the external and internal geometry of the mesh was accomplished by defining 48 of these regions. The regions were divided into six sets of eight with each set lying between a pair of parallel planes oriented perpendicular to the $x$-axis. In any of these sets of regions, the three outer regions (i.e. those furthest from the $x$-axis) represented the silver sheath (material type 1 ) and the five inner ones were part of the superconducting core (material type 2). The mesh generator input file for the first pass can be found in the file davies/First_pass/MF/Two_mat/mf.mesh. Note that due to an alteration of the mesh generator made during the course of this project, the material type of each region must be declared explicitly in the mesh generator input file. This declaration is made on the line preceding the specification of the number of divisions per side. The material type is used by mesh3d.v2 to generate a file filename.mat that contains a list of all elements in the mesh that are not of material type 1. This list can be used in the input file for ISAIAH as described in the next subsection.

It is particulariy important to note the manner in which the interface surface, 9, was declared in mf.mesh. Definition of surface 9 was ambiguous since it could equivalently be declared as the outward facing surface of the superconducting core or as the inward facing surface of the outer sheath. In order to ensure the proper definition of the surface element normal vector directions, the former declaration was used. 


\section{A.2 ISAIAH Input File}

The input file used for the first pass rolling simulation was “ davies /First_pass /MF /Two_mat $/ M F 8_{-} 6_{-} 9 / m f . i i$. In this section only highlights of the input file are discussed. For a comprehensive discussion of ISAIAH input files and the various options available in the program see Dewhurst et. al. [1].

The input file, mf.ii, instructed the program to initiate an Eulerian solution to the viscoplastic flow problem. There were two materials in the problem with the superconducting core labeled material 2. Thus, on line five of $m f . i i$ the variable notone was set to 228 , the number of elements of material type 2 (superconducting core). The declaration of notone was followed by a list of the core elements and their material types. The list of these elements as well as their respective material types was written by the mesh generator to the file $m f . m a t$. This list was then copied directly to the input file.

Following the element list is the declaration of the properties for the two materials. The density and Poisson's ratio for each material were $2707 \mathrm{~kg} / \mathrm{m}^{3}$ and 0.499995 , respectively ${ }^{2}$. Nonlinear material behavior was simulated by a simple non-evolving power law model as described in the previous section on material behavior.

Boundary conditions were defined on each of the ten surfaces of the mesh. On the inlet and outlet cross-sections, the material was defined to have zero $y$-and z-velocity. On the symmetry surfaces 2 and 4 , the material had zero $z$ - and y-velocity. Surfaces 3,8 and 6 were the outer traction-free outer surfaces. The internal surfaces 9 and 10 were also declared to be traction free. This apparently non-physical definition simply ensured that there were no alterations made to the calculated force matrices during the simulation; thus the solution was unaffected. A sticking condition was assumed

\footnotetext{
${ }^{2}$ Parameter values provided by Dave Korzekwa at Los Alamos
} 
between the roll and the workpiece, and therefore a tangential velocity of $5 \mathrm{~mm} / \mathrm{s}$ was imposed on the roll contact surface.

Three surfaces were designated for correction by setting the variable nsidfs to 3. These surfaces were the external traction-free surfaces 6 and 8 and the internal surface 9 . Surfaces 8 and 9 were corrected along the surface normals while surface 6 was corrected in the $z$-direction. The boundary conditions for the surface correction problem were defined as follows.

1. Surface 9 is fixed to surface 10 .

2. Surface 8 is fixed to surfaces 3 and 5 .

3. Surface 6 is fixed to surface 5 .

Finally, to avoid possible high-frequency spatial surface oscillations, the positions of the midside nodes were defined to be the average of the corrected corner node locations.

\section{A.3 Running the Frontal Program and ISAIAH}

Before running ISAIAH, the frontal program described in the manual [1] was run. The input file used for the frontal program was -davies /First_pass /MF /Two_mat /MF8_6_98/fi.mf.

After running the frontal program, ISAIAH was executed. The version used for these simulations was affe.v1. The version affe.v2, that contains additional features such as mesh smoothing between surface corrections, is currently under development. 


\section{A.4 Generation of Mesh for the Second Pass}

The program meshpost ${ }^{3}$, was used to generate meshes for successive passes. This was accomplished by mapping the mesh nodal points to new positions that were determined from the roll reduction information and the final, corrected cross-section from the previous pass. Because the new mesh was created by mapping the previous mesh nodal points, the new mesh and the old mesh had identical nodal point and element numbering and equivalent connectivity. To eliminate element distortions caused by the mapping, the program concluded it's execution by smoothing the nodal point coordinates.

Before running meshpost, a listing of the final corrected nodal point coordinates calculated during the previous pass was placed in an input file. This information was extracted from the.$a d x$ file. For the case discussed in this report, the final 1693 (number of nodal points) lines of $m f . a d x$ were extracted and written to the file mf.final. This file was then used as input to meshpost.

In addition to the file containing the final geometry from the previous pass, meshpost also required a prototype mesh. The prototype provided a reference numbering scheme for the mesh nodal points used in the mapping and smoothing routines. An example of a prototype mesh is given in davies /First_pass /MF /Two_mat /MF8_6 $\sigma_{-} 9$ $/ N e x t \_m e s h / r . p t$. The input file for this mesh was pt.mesh. The prototype consisted of a simple mesh of constant circular cross section, where the regions, the number of elements, and the connectivity were identical to those of $\mathrm{mf.mesh}$.

After creating the two input files, meshpost was executed. An example session of meshpost follows.

prototype mesh

\footnotetext{
${ }^{3}$ Meshpost was written by D. E. Boyce
} 


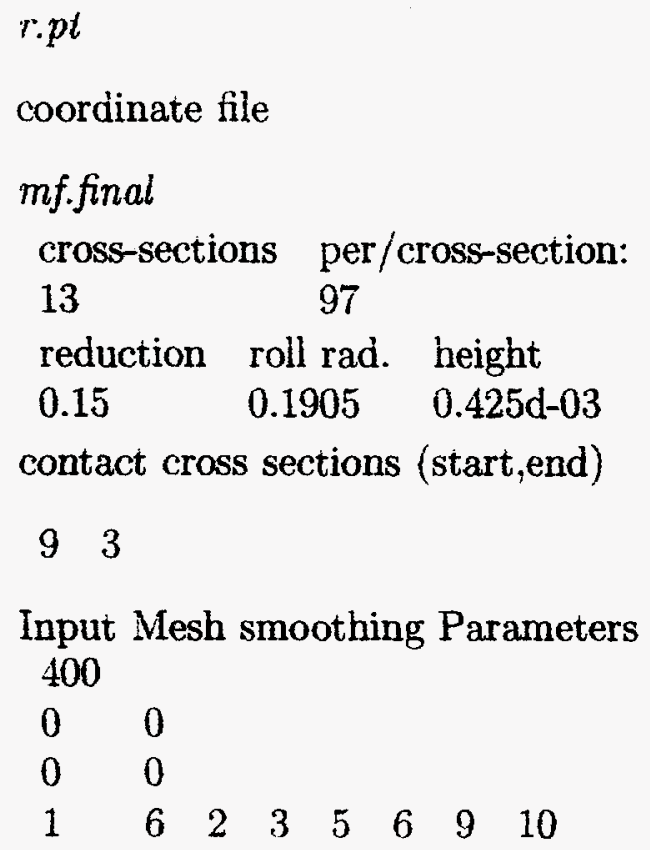

Prompt 1 requests the name of the file containing the corrected mesh geometry from the previous pass, mf.final for this case. Prompt 2 requests the name of the prototype file, r.pt.

Prompt 3 requests two numbers. The first is the number of cross-sections parallel to the $(y, z)$-plane that separate the various groups of elements in the mesh. The second is the number of nodes lying on each cross-section. This information allows the program to take advantage of the particular mesh geometry used in these simulations. The mesh is divided into groups of elements that are separated by a set of planes that are oriented perpendicular to the $x$-axis. For the mesh r.mf there are 13 of these planes and on each plane there are 97 nodal points.

Prompt 4 requests the roll reduction fraction $f$ ( 0.15 for this case), the roll radius $(0.01905 \mathrm{~m})$, and the final $z$-direction thickness of the mesh for the previous pass. For the first pass, this thickness is $15 \%$ less than the initial value of $0.5 \mathrm{~mm}$, or 0.425 $\mathrm{mm}$. 
Prompt 5 requests information about the number of mesh cross-sections that intersect the roll surface. The cross-sections are labeled from outlet to inlet so that the outlet is cross-section number 1 and the inlet is cross-section number 13. Thus cross-sections 3 through 9 intersect the roll surface. As shown, the proper format for input in this case is " 9 ".

Prompt 6 requests the label of the roll contact surface (global surface 5) and the post contact surface (global surface 6) as defined in the mesh generator.

The final prompt requests a number of input parameters that are used in the mesh smoothing routines. The first is the number of smoothing passes, nsmooth. A value of 400 works well. Next there are three sets of input parameters that control mesh smoothing in the $x, y$ and $z$ directions, respectively. The first number in each set is a smoothing flag. If the value of the flag is 1 , smoothing will be done. If it is zero, smoothing will not be done. The second parameter in each set designates the number of global mesh surfaces to be held fixed during the smoothing. If this number is nonzero it will be followed by a list of those surfaces to be held fixed. This set of input parameters must be repeated for each direction, $x, y$ and $z$. Therefore, in the example the program is instructed to smooth the mesh in the z-direction only, while holding nodal points on the 6 designated global surfaces fixed. Generally, this type of z-coordinate smoothing is sufficient to generate an acceptable mesh for the next pass.

Meshpost created a mesh file r.newmesh for use in the next reduction pass. An example for the first pass can be found in “davies/ First_pass/ MF/ Two_mat $/ M F 8_{-} 6_{-} 9 / N e x t_{-} m e s h$ where the output from the first pass has been used to create a mesh for the second pass. 


\section{A.5 Location of Information for Further Rolling Passes}

A total of six rolling passes were run using the mesh r.mf. The results are located in directories davies $/ *$ _pass /MF where the * represents the words First, Second, Third etc. The initial meshes for the first and second passes were generated manually, while the meshes for the remaining four passes were generated by meshpost.

\section{References}

[1] T. B. Dewhurst and P. R. Dawson. A finite element program for viscoplastic flow and heat transfer in three dimensions, version 2.0, 1985. Report to the Bethlehem Steel Corporation Homer Research Laboratory. 